\title{
MC DS/SFH-CDMA Systems for Overlay Systems
}

\author{
Jiangzhou Wang, Senior Member, IEEE and Hu Huang
}

\begin{abstract}
In this paper, we introduce the slow frequency hopping (SFH) technique to the multicarrier (MC) code-division multiple-access (CDMA) systems for overlay situations. Using lower chip rate, which results in a narrower spectrum for each carrier and hopping the signal in frequency, the MC direct sequence (DS)/SFH system achieves better performance than the MC DS CDMA system in most cases, especially when the bandwidth of the narrowband interference (NBI) is narrower than one subchannel. It also exhibits a stable performance against the variations of the NBI location and bandwidth. When there is no NBI, the two systems perform approximately the same. The evaluation is performed over a frequency selective Rayleigh fading channel, with both Gaussian approximation and Monte Carlo simulation.
\end{abstract}

Index Terms-Code-division multiple-access, multicarrier systems, frequency hopping, narrowband interference suppression.

\section{INTRODUCTION}

$\mathbf{M}$ ULTICARRIER (MC) code-division multiple-access (CDMA), which is robust to multipath fading and effective in narrowband suppression, has drawn a lot of concern in recent years. Various schemes of MC CDMA systems have been proposed in recent years, which can be categorized into two groups. The first group is orthogonal multicarrier [1] in which different adjacent subcarriers are orthogonally overlapped. The other group is disjoint multicarriers [2], [3] in which all subcarriers are not overlapped. The second group contains two subtypes: one is that different data streams modulate different carriers and another one is that all carriers are modulated by the same data stream. The system proposed in this paper belongs to the latter.

Among the various advantages of MC CDMA techniques, its desirable capability of narrowband suppression is of most interest to us. It has been shown in [3] that the MC direct sequence (DS) CDMA systems are ingenious in suppressing narrowband interference (NBI) via frequency diversity. Since the interference is usually limited in some of the subchannels, the decision can be made based on the remaining "clean" ones. Thus, there is no need for either a suppression filter at the receiver or a notch filter at the transmitter. However, the system performance varies noticeably for different interference center frequencies, because

Manuscript received July 15, 2000; revised June 28, 2001; accepted July 18, 2001. The editor coordinating the review of this paper and approving it for publication is V. V. Veeravalli. This work was supported by the Research Grant Council (RGC) of the Hong Kong Government.

$\mathrm{J}$. Wang is with the Department of Electrical and Electronic Engineering, University of Hong Kong, Kowloon, Hong Kong (e-mail: jwang@eee.hku.hk).

H. Huang is with the Department of Electrical and Electronic Engineering, University of Hong Kong, Kowloon, Hong Kong and also with the Department of Electrical and Computer Engineering, University of Maryland, College Park, MD 20742 USA.

Publisher Item Identifier 10.1109/TWC.2002.800543 the division of the channel is determined by its coherence bandwidth to ensure independent fading in each subchannel, irrespective to the interference. Once the interference affects two or more subchannels, although its bandwidth does not exceed one subchannel, the performance degrades much from that when the interference is limited in one subchannel.

To relieve such dependence, each subchannel is further divided into multiple frequency slots in the proposed MC DS/SFH systems. Different to the system proposed in [3], the transmitted signal occupies only one slot of each subchannel and all carriers are uniformly hopped in frequency. The proposed systems show their advantages when the interference only overlays parts of the slots in a subchannel, because an extra "clean" diversity branch is obtained when the signal is hopped to the slot unaffected and the average bit error rate is reduced accordingly. Although the spreading gain of each carrier is reduced by the factor of the number of frequency slots, the average cochannel user number is also reduced by the same factor. Therefore, MC DS/SFH systems have approximately the same multiaccess capability as the MC DS system. The performance of the proposed system is studied in a multipath Rayleigh fading channel and compared with that of MC DS CDMA systems, given the same information rate and the same system bandwidth.

The remainder of the paper is organized as follows. In Section II, the system models are described. In Section III, the performance of the MC DS/SFH-CDMA systems in terms of average bit-error rate (BER) is analyzed. Numerical results are given in Section IV. Finally, the conclusions are presented in Section V.

\section{SYSTEM MODEL}

In the proposed MC DS/SFH systems, the total bandwidth $B_{T}$ is divided into $M$ equi-width subchannels of bandwidth $B_{M}=$ $B_{T} / M$ and central frequency

$$
f_{m}=f_{c}+B_{T} / M \cdot[m-(M+1) / 2], \quad m=1, \ldots, M
$$

where $f_{c}$ is the central frequency of the total system bandwidth. We adopt the same assumption as in [3] to choose $M$ : each carrier of the system has only one resolvable path; all carriers are subject to independent fading. The low pass equivalent impulse response of the $m$ th subchannel for the $k$ th user is then given by

$$
h_{k, m}(t)=\beta_{k, m} e^{j \mu_{k, m}} \delta\left(t-\tau_{k}\right)
$$

where $\left\{\beta_{k, m}\right\}$ are independent identically distributed (i.i.d.) Rayleigh distributed with $E\left[\beta_{k, m}^{2}\right]=2 \rho$ for different $k$ and $m$, and the phases $\left\{\mu_{k, m}\right\}$ are uniformly distributed in $[-\pi, \pi) . \tau_{k}$ is the relative delay of the $k$ th user and is randomly distributed in $[0, T)$, where $T$ is the bit interval. 


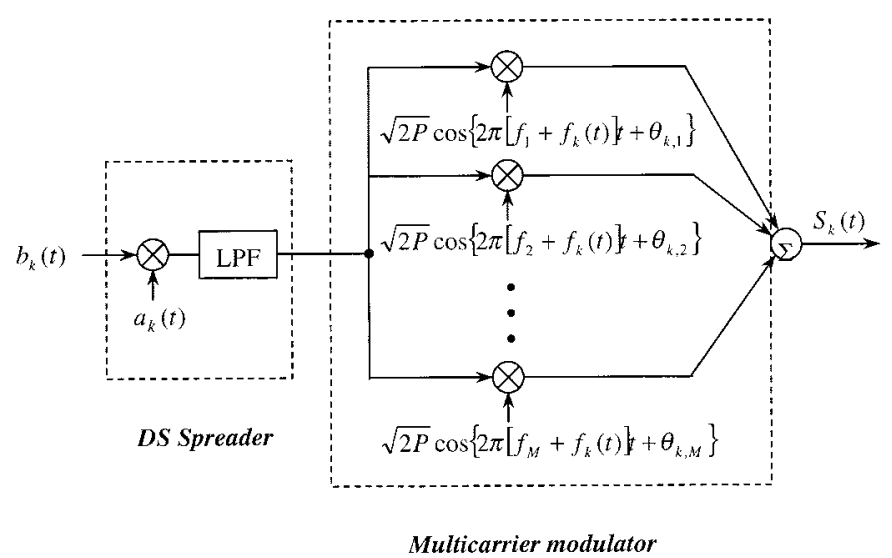

Fig. 1. Transmitter structure of the MC DS/SFH system.

Each subchannel is further divided into $Q$ frequency slots, each with bandwidth $B_{s}=B_{M} / Q$, where $Q$ is the number of available hopping frequencies. The $M$ carriers of the $k$ th user are uniformly hopped by the same hopping pattern, $f_{k}(t)$, which takes values randomly from the frequency set

$$
f^{(q)}=\frac{B_{M}}{Q}[q-(Q+1) / 2], \quad q=1, \ldots, Q
$$

in each hopping interval and is independent to the hopping pattern of other users.

The transmitter for the $k$ th user is shown in Fig. 1, where $b_{k}(t)$ is the binary data sequence with bit rate $R_{b}=1 / T, a_{k}(t)$ is the random signature sequence with chip duration $T_{c}=T / N$, where $N$ is the spreading gain of one carrier. Strictly speaking, the chip waveform should be defined by a low-pass shaping filter (i.e., square-root raised cosine filter). However, for simple analysis, a rectangular chip waveform is assumed. Since the side lobes of the signal power spectrum is very small compared with its main lobe, the transmit signal is assumed to be approximately bandlimited by its null-to-null bandwidth $2 / T_{c}=B_{s}$ to ensure that there is no overlap between transmitted signals of two users in frequency if they take different hopping frequencies. The total processing gain is then $N_{T}=B_{T} /(2 / T)=\mathrm{MQN}$. The transmitted signal of the $k$ th user can be written as

$$
S_{k}(t)=\operatorname{Re}\left[\sqrt{2 P} \sum_{m=1}^{M} C_{k, m}(t)\right]
$$

where $P$ is the transmitted power of each carrier and

$$
C_{k, m}(t)=b_{k}(t) a_{k}(t) \exp \left\{j 2 \pi\left[f_{m}+f_{k}(t)\right] t+j \theta_{k, m}\right\}
$$

and $\theta_{k, m}$ is the random phase introduced. Note that when $Q=$ 1 , the system is identical to the MC DS CDMA system. The power spectrum densities of the transmitted signal are shown in Fig. 2, where the number of subchannels $M=4$.

The NBI is characterized as band-limited white Gaussian noise with the double-sided power spectral density $\eta_{J} / 2$ and bandwidth $B_{J}$, as shown in Fig. 2. Its power spectral density is given by

$$
S_{J}(f)= \begin{cases}\frac{\eta_{J}}{2}, & f_{J}-\frac{B_{J}}{2} \leq|f| \leq f_{J}+\frac{B_{J}}{2} \\ 0, & \text { else }\end{cases}
$$

where $f_{J}$ is the central frequency of the interference.
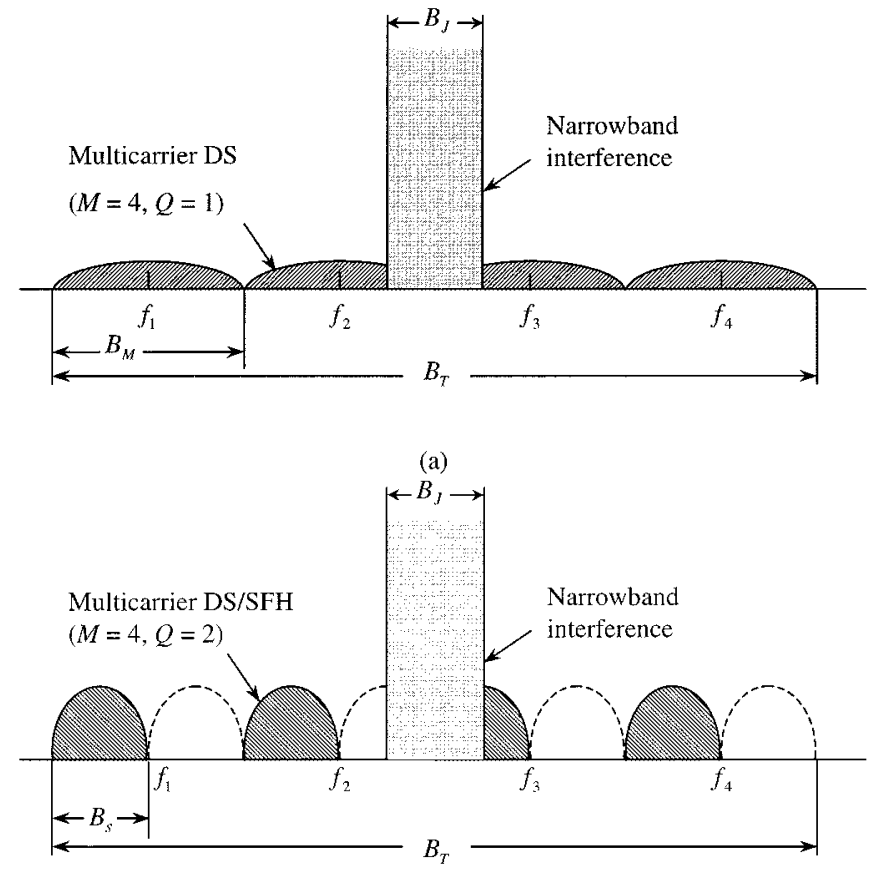

(b)

Fig. 2. Power spectrum densities of transmitted signals. (a) MC DS system and the NBI. (b) MC DS/SFH system and the NBI.

The ratio of the interference bandwidth to the system bandwidth is defined as

$$
p=\frac{B_{J}}{B_{T}},
$$

the ratio of the offset of the interference central frequency to half of the system bandwidth is defined as

$$
\delta=\frac{\left|f_{J}-f_{c}\right|}{B_{T} / 2}
$$

with $0 \leq \delta<1$ and the ratio of the interference power to the signal power is defined as

$$
J / S=\frac{P_{J}}{E_{b} / T}
$$

where $P_{J}=\eta_{J} B_{J}$ is the interference power, $E_{b}$ is the total received signal energy per bit.

In time domain, the interference can then be written as

$$
J(t)=\operatorname{Re}\left\{\left[J_{c}(t)+j J_{s}(t)\right] \exp \left(j 2 \pi f_{J} t\right)\right\}
$$

where both $J_{c}(t)$ and $J_{s}(t)$ are independent low-pass processes with $E\left[J_{c}^{2}(t)\right]=E\left[J_{s}^{2}(t)\right]=P_{J}$.

The received signal can be written as

$$
\begin{aligned}
r(t)= & \operatorname{Re}\left[\sqrt{2 P} \sum_{k=1}^{K} \sum_{m=1}^{M} C_{k, m}(t) * h_{k, m}(t)\right]+J(t)+n(t) \\
= & \operatorname{Re}\left[\sqrt{2 P} \sum_{k=1}^{K} \sum_{m=1}^{M} \beta_{k, m} e^{j \mu_{k, m}} C_{k, m}\left(t-\tau_{k}\right)\right] \\
& +J(t)+n(t)
\end{aligned}
$$




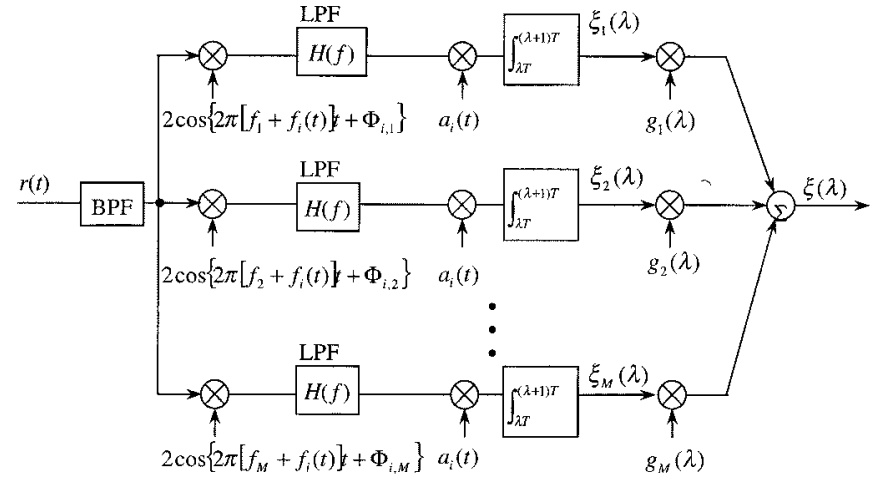

Fig. 3. Block diagram of the receiver for user $i$,

where $*$ stands for convolution, $n(t)$ is the additive white Gaussian noise with one-sided spectral power density of $N_{0}$.

The receiver for the $i$ th user (the reference user) is shown in Fig. 3, which consists of $M$ correlators and a maximal ratio combiner. A LPF with frequency response $H(f)=1$ and 0 in $\left[-B_{s} / 2, B_{s} / 2\right]$ and otherwise, respectively, is employed for each carrier to eliminate the effects of NBI components out of the current slot.

\section{PeRformance AnAlysis}

In the presence of NBI, the performance of a super-high frequency (SFH) system depends on the instantaneous value of its hopping frequency. Conditioned on $f_{i}(\lambda T)=f^{(q)}$, the output of the correlator for the $m$ th carrier in the $\lambda$ th symbol interval is given by

$$
\begin{array}{r}
\xi_{m}(\lambda)=\int_{\lambda T}^{(\lambda+1) T}\left\{2 r(t) \cos \left[2 \pi\left(f_{m}+f^{(q)}\right) t+\Phi_{i, m}\right]\right. \\
* h(t)\} a_{i}(t) d t
\end{array}
$$

where $\Phi_{i, m}=\theta_{i, m}+\mu_{i, m}$ and $h(t)$ is the impulse response of the ideal low-pass filter. Neglecting the high frequency terms, (11) reduces to

$$
\xi_{m}(\lambda)=D_{m}+N_{m}+J_{m}+I_{m}
$$

where $D_{m}=\sqrt{2 P} T \beta_{i, m} b_{i}^{(\lambda)}$ is the desired signal component, where $b_{i}^{(\lambda)}$ stands for the $\lambda$ th bit of the reference user with $b_{i}^{(\lambda)}=$ 1 or -1 .

$N_{m}$ is the noise term and given by

$$
\begin{array}{r}
N_{m}=\int_{\lambda T}^{(\lambda+1) T}\left\{2 n(t) \cos \left[2 \pi\left(f_{m}+f^{(q)}\right) t+\Phi_{i, m}\right]\right. \\
* h(t)\} a_{i}(t) d t .
\end{array}
$$

It is easy to shown that $N_{m}$ is a Gaussian random variable with zero mean and variance $\sigma_{N}^{2}$ equal to $N_{0} T$.

$J_{m}$ is due to the NBI and can be written as

$$
J_{m}=\int_{\lambda T}^{(\lambda+1) T} \hat{J}_{m}(t) a_{i}(t) d t
$$

where

$$
\hat{J}_{m}(t)=2 J(t) \cos \left[2 \pi\left(f_{m}+f^{(q)}\right) t+\Phi_{i, m}\right] * h(t) .
$$

The power spectral density of $\hat{J}_{m}(t)$ is given by

$$
\begin{aligned}
S_{J_{m}}(f)= & \left\lfloor S_{J}\left(f+f_{m}+f^{(q)}\right)\right. \\
& \left.+S_{J}\left(f-f_{m}-f^{(q)}\right)\right\rfloor H^{2}(f) \\
= & S_{m}(f)+S_{m}(-f)
\end{aligned}
$$

where $H(f)=1$ when $|f| \leq B_{s} / 2$ and $H(f)=0$, otherwise

$$
\begin{aligned}
S_{m}(f) & =S_{J}\left(f+f_{m}+f^{(q)}\right) H^{2}(f) \\
& = \begin{cases}\eta_{J} / 2, & f_{l} \leq f \leq f_{h} \\
0, & \text { else. }\end{cases}
\end{aligned}
$$

In (15b), $f_{l}$ and $f_{h}$ are defined as

$$
\left\{\begin{array}{l}
f_{h}=\min \left[f_{J}+B_{J} / 2-f_{m}-f^{(q)}, B_{s} / 2\right] \\
f_{l}=\max \left[f_{J}-B_{J} / 2-f_{m}-f^{(q)},-B_{s} 2\right] .
\end{array}\right.
$$

The autocorrelation function of $\hat{J}_{m}(t)$ is then given by

$$
\begin{aligned}
\hat{R}_{J_{m}}(\tau) & =E\left[\hat{J}_{m}(t) \hat{J}_{m}(t+\tau)\right] \\
& =\eta_{J} \frac{\sin \left(\pi B_{J_{m}} \tau\right)}{\pi \tau} \cos \left(2 \pi \Delta_{J_{m}} \tau\right)
\end{aligned}
$$

where

$$
\left\{\begin{array}{l}
B_{J_{m}}=\max \left(f_{h}-f_{l}, 0\right) \\
\Delta_{J_{m}}=\left(f_{h}+f_{l}\right) / 2
\end{array} .\right.
$$

Since $J(t)$ is a Gaussian process, $J_{m}$ is also a zero-mean Gaussian random variable. Its variance $\sigma_{m}^{2}(q)$, conditioned on $q$, is given by

$$
\begin{aligned}
\sigma_{m}^{2}(q)= & \int_{\lambda T}^{(\lambda+1) T} \int_{\lambda T}^{(\lambda+1) T} E\left[\hat{J}_{m}\left(t_{1}\right) \hat{J}_{m}\left(t_{2}\right)\right] \\
& \cdot E\left[a_{i}\left(t_{1}\right) a_{i}\left(t_{2}\right)\right] d t_{1} d t_{2} \\
= & \int_{\lambda T}^{(\lambda+1) T} \int_{\lambda T}^{(\lambda+1) T} \hat{R}_{J_{m}}\left(t_{1}-t_{2}\right) \\
& \times R_{i}\left(t_{1}-t_{2}\right) d t_{1} d t_{2} \\
= & \left.\int_{-T}^{T} \hat{R}_{J_{m}} \tau\right) R_{i}(\tau)(T-|\tau|) d \tau
\end{aligned}
$$

where $R_{i}(\tau)$ is the autocorrelation function of $a_{i}(t)$ and triangular in $\left(-T_{c}, T_{c}\right)$. Substituting $\hat{R}_{J_{m}}(\tau)$ with (16a), $B_{J_{m}}$ and $\Delta_{J_{m}}$ with (16b), and $T_{c}$ with $2 / B_{s}$, (17a) can be rewritten as

$$
\sigma_{m}^{2}(q)=P_{J} T^{2} X(m, q)
$$

where (17c) and (17d), are shown at the bottom of the next page.

$I_{m}=\sum_{k=1}^{K_{h}} I_{k, m}$ is the total multiple access interference (MAI) term, where $K_{h}$ is the number of nonreference users that collide with the reference user in frequency $\left(f_{k}(\lambda T)=f^{(q)}\right)$ and has a binomial distribution with probability density function given by

$$
P_{H}\left(K_{h}\right)=\left(\begin{array}{c}
K-1 \\
K_{h}
\end{array}\right)\left(P_{h}\right)^{K_{h}}\left(1-P_{h}\right)^{K-K_{h}-1}
$$

where $P_{h}$ is the probability that a nonreference user hits the reference user and approximates to $1 / Q$ for slow frequency hopping [6]. 
$I_{k, m}$ is due to the signal of the $k$ th user $(k \neq i)$ and given by

$$
\begin{aligned}
I_{k, m}= & \sqrt{2 P} \beta_{k, m} \cos \left(\Phi_{k, m}-\Phi_{i, m}\right) \\
& \times \int_{\lambda T}^{(\lambda+1) T}\left[b_{k}\left(t-\tau_{k}\right) a_{k}\left(t-\tau_{k}\right) * h(t)\right] a_{i}(t) d t \\
= & \sqrt{2 P} \beta_{k, m} \cos \left(\Phi_{k, m}-\Phi_{i, m}\right) \\
& \times \int_{\lambda T}^{(\lambda+1) T} b_{k}\left(t-\tau_{k}\right) a_{k}\left(t-\tau_{k}\right) a_{i}(t) d t
\end{aligned}
$$

where $\Phi_{k, m}=\theta_{k m}+\mu_{k, m}-2 \pi f_{k}(\lambda T) \tau_{k} . I_{k, m}$ passes through the LPF of $h(t)$ without any distortion or attenuation since the bandwidth of the LPF is the same as that of $I_{k, m}$.

It is easy to prove that $\left\{I_{k, m}\right\}$ are identically distributed but uncorrelated for different $k$. When $K_{h}$ is large, one can use the Gaussian approximation [8] and treat $I_{m}$ as a random Gaussian variable. Its variance, conditioned on $K_{h}$, is given by

$$
\sigma_{I}^{2}\left(K_{h}\right)=4 K_{h} P \rho T^{2} /(3 N)
$$

The SNR of $\xi_{m}(\lambda)$, conditioned on $\beta_{i, m}, K_{h}$ and $q$, is defined as

$$
\begin{aligned}
\left.\gamma_{m}\right|_{\beta_{i, m}, K_{h}, q} \\
=\frac{E^{2}\left[\xi_{m}(\lambda) \mid \beta_{i, m}\right]}{2 \operatorname{Var}\left[\xi_{m}(\lambda) \mid K_{h}, q\right]} \\
=\frac{2 P T^{2}\left(\beta_{i, m}\right)^{2}}{2\left[\sigma_{I}^{2}\left(K_{h}\right)+\sigma_{m}^{2}(q)+\sigma_{N}^{2}\right]} \\
=\frac{\left(\beta_{i, m}\right)^{2}}{2 \rho}\left[2 K_{h} /(3 N)+\frac{P_{J} T^{2} X(m, q)+N_{0} T}{E_{b} T / M}\right]^{-1} \\
=\frac{\left(\beta_{i, m}\right)^{2}}{2 \rho}\left[2 K_{h} /(3 N)+M J / S \cdot X(m, q)\right. \\
\left.\quad+M\left(E_{b} / N_{0}\right)^{-1}\right]^{-1}
\end{aligned}
$$

where $E_{b}=2 M \rho P T$ is the total received signal energy per bit.

By definition, $\left\{N_{m}\right\}$ in (13) are i.i.d. It is also easy to verify that $\left\{I_{m}\right\}$ and $\left\{J_{m}\right\}$ are uncorrelated for different $m$. Therefore, $\left\{\xi_{m}(\lambda)\right\}$ are mutually uncorrelated. To maximize the SNR of the final decision variable, each $\xi_{m}(\lambda)$ is weighted by a tap gain [7]

$$
g_{m}(\lambda)=\frac{E\left\lfloor b_{i}^{(\lambda)} \xi_{m}(\lambda) \mid \beta_{i, m}\right\rfloor}{\operatorname{Var}\left[\xi_{m}(\lambda) \mid K_{h}, q\right]}
$$

where, we assume that the fading is slow enough that the conditional mean and variance of $\xi_{m}(\lambda)$ can be accurately estimated. The final decision variable is then given by

$$
\xi(\lambda)=\sum_{m=1}^{M} g_{m}(\lambda) \xi_{m}(\lambda)
$$

and its instantaneous SNR, conditioned on $\left\{\beta_{i, m}\right\}, K_{h}$ and $q$, is given by

$$
\begin{aligned}
\left.\gamma_{b}\right|_{\left\{\beta_{i, m}\right\}, K_{h}, q}= & \frac{E^{2}\left[\xi(\lambda) \mid\left\{\beta_{i, m}\right\}\right]}{2 \operatorname{Var}\left[\xi(\lambda) \mid K_{h}, q\right]}=\left.\sum_{m=1}^{M} \gamma_{m}\right|_{\beta_{i, m}, K_{h}, q} \\
= & \sum_{m=1}^{M} \frac{\left(\beta_{i, m}\right)^{2}}{2 \rho}\left[2 K_{h} /(3 N)+M J / S\right. \\
& \left.\cdot X(m, q)+M\left(E_{b} / N_{0}\right)^{-1}\right]^{-1} .
\end{aligned}
$$

The conditional BER takes the simple form

$$
P_{e}\left(\gamma_{b}\right)=\frac{1}{\sqrt{2 \pi}} \int_{\sqrt{2 \gamma_{b}}}^{\infty} e^{-x^{2} / 2} d x .
$$

To get the final average BER $P_{e}$, we first find the BER $\left.P_{e}\right|_{K_{h}, q}$, conditioned on $K_{h}$ and $q$, which can be obtained by averaging $P_{e}\left(\gamma_{b}\right)$ over the statistics of $\left\{\beta_{i, m}\right\}$. The detailed derivation of $\left.P_{e}\right|_{K_{h}, q}$ is shown in Appendix A. Assuming all hopping frequencies appear with equal probability, the final average BER is given by

$$
P_{e}=\frac{1}{Q} \sum_{q=1}^{Q}\left[\left.\sum_{K_{h}=0}^{K-1} P_{e}\right|_{K_{h}, q} \cdot P_{H}\left(K_{h}\right)\right]
$$

where $P_{H}\left(K_{h}\right)$ is the probability density function (pdf) of $K_{h}$, given by (18), and $\left.P_{e}\right|_{K_{h}, q}$ is given by (A5) when all diversity branches have the same SNR or (A8) when the SNRs of different branches are not equal.

In case $K_{h}$ is small, the BER obtained by Gaussian approximation may not be accurate enough. However, small values of $K_{h}$ appear with small hit probability and small conditional BER. Therefore, their contributions to the final average BER are not significant. In the numerical section, the results of Gaussian approximation are verified by Monte Carlo simulations, which reveal the applicability of the Gaussian approximation in most situations. The simulation model is described in Appendix B.

\section{NUMERICAL RESULTS}

In this section, we consider the MC DS/SFH system $(N=$ $63, Q=2$ ), and compare its performance to that of the MC DS system $(N=127, Q=1)$ for the same system bandwidth.

$$
X(m, q)=\frac{1}{p N_{T}} U\left(\begin{array}{c}
\min [M Q(\delta+p)-Q(2 m-M-2)-2 q+1,1], \\
\max [M Q(\delta-p)-Q(2 m-M-2)-2 q+1,-1]
\end{array}\right)
$$

where

$$
U(u, v)= \begin{cases}\int_{0}^{1} \frac{\sin (2 \pi u \tau)-\sin (2 \pi v \tau)}{2 \pi \tau}(1-\tau)(1-\tau / N) d \tau, & u>v \\ 0, & u \leq v\end{cases}
$$




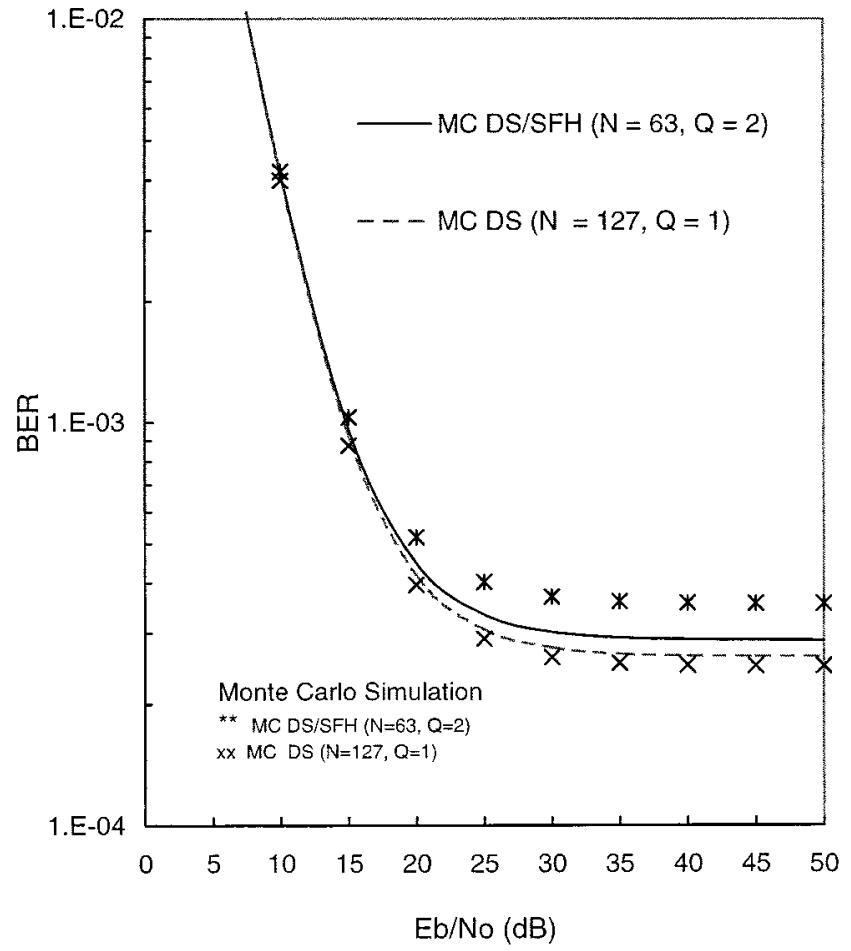

Fig. 4. BER performance with respect to $E_{b} / N_{0}$ in the absence of NBI ( $K=$ $50, J / S=-\infty \mathrm{dB})$.

Pseudonoise (PN) sequences applied in Monte Carlo simulations are Gold sequences. The set of sequences of period 127 is generated by the polynomial 41567 , and the set of sequences of period 63 is generated by the polynomial 13055 . The initial loadings are given in [9] to generate a class of codes known as auto-optimal with least sidelobe energy (AO/LSE). In all cases, $M=4$ and $K=50$ if not specified.

In the absence of NBI, the average SNRs of all carriers are identical and $\left.P_{e}\right|_{K_{h}, q}$ for both systems can be given by (A5)

$$
\left.P_{e}\right|_{K_{h}, q}=\mu^{4} \sum_{i=0}^{3}\left(\begin{array}{c}
3+i \\
i
\end{array}\right)(1-\mu)^{i}
$$

where $\mu=\left\lfloor 1-\sqrt{r_{0} /\left(1+r_{0}\right)}\right\rfloor / 2$, while $r_{0}$ is the common SNR given by

$$
r_{0}=\left[2 K_{h} /(3 N)+4\left(E_{b} / N_{0}\right)^{-1}\right]^{-1} .
$$

The BERs against $E_{b} / N_{0}$ of the two systems without NBI are given in Fig. 4, obtained by both Gaussian approximation and Monte Carlo simulation. It is shown that the two systems have almost the same performance, although the BER of the MC DS/SFH system is slightly higher when $E_{b} / N_{0}$ is large. This is predictable because $r_{0}$ is a concave function of $K_{h}$, therefore, the SNR per carrier averaged over $K_{h}$

$$
\begin{aligned}
E\left[r_{0}\right] & \leq\left[\frac{2 E\left[K_{h}\right]}{3 N}+4\left(\frac{E_{b}}{N_{0}}\right)^{-1}\right]^{-1} \\
& =\left[\frac{2(K-1)}{3 N_{T} / 4}+4\left(\frac{E_{b}}{N_{0}}\right)^{-1}\right]^{-1}
\end{aligned}
$$

while the right hand of the inequality is the average SNR per carrier for the MC DS system. Note that the analytical results

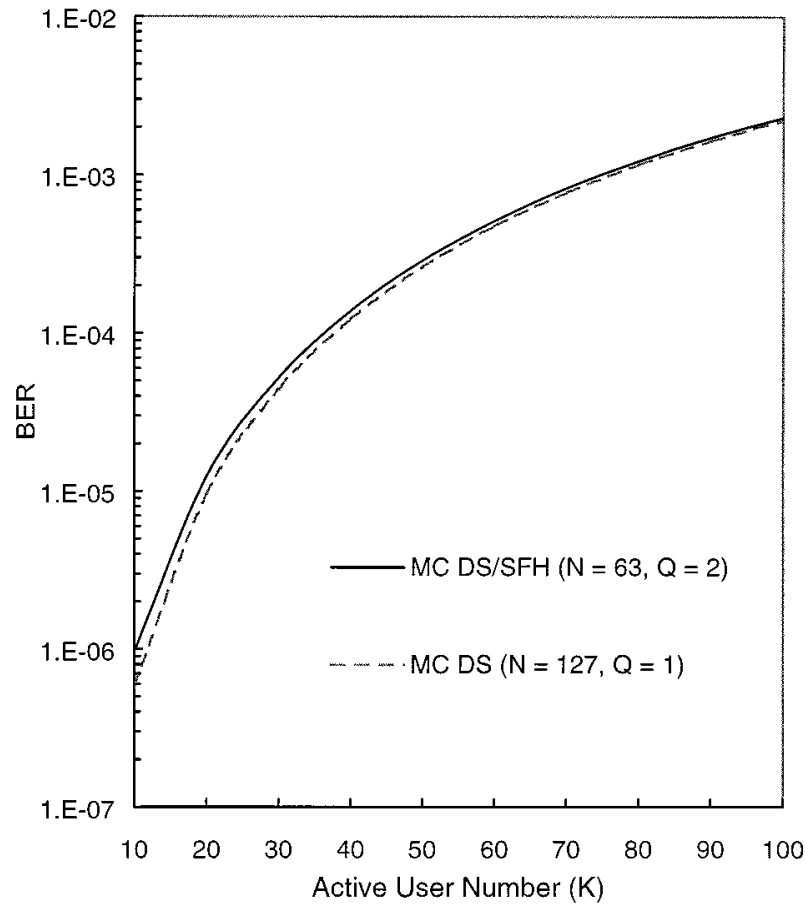

Fig. 5. Comparison of the MC DS/SFH system and the MC DS system for different system loads $\left(J / S=-\infty \mathrm{dB}, E_{b} / N_{0} \rightarrow \infty\right)$

of MC DS/SFH are optimistic compared with its simulation results. This is because Gaussian approximation is optimistic for MC DS/SFH due to small number of instantaneous users which have the same frequencies with the reference user.

In Fig. 5, the asymptotic BERs $\left(E_{b} / N_{0} \rightarrow \infty\right)$ of the MC DS/SFH and the MC DS systems are plotted for different system loads. It is shown that the differences of the performance between the two systems diminish as the number of active user increases.

Next, we compare their performance in the presence of NBI with bandwidth $B_{J}$ equal to one subchannel $(p=0.25)$ and central frequency equal to $f c(\delta=0)$. For the MC DS system, the two middle carriers are overlaid by the interference. Their average SNRs are identical and given by

$$
r_{1}=\left\{2 K_{h} /(3 N)+4\left[\frac{J / S \cdot U(1,0)}{0.25 N_{T}}+\left(E_{b} / N_{0}\right)^{-1}\right]\right\}^{-1}
$$

while the SNRs of the other two carriers free of interference are equal to $r_{0}$. Therefore, its $\left.P_{e}\right|_{K_{h}, q}$ take the same formula in the two systems and given by (A10)

$$
\begin{aligned}
\left.P_{e}\right|_{K_{h}, q}=A_{10}^{2} \mu_{0}^{2}\left(3-2 \mu_{0}\right)+2 A_{10}^{2} A_{01} \mu_{0} \\
+2 A_{01}^{2} A_{10} \mu_{1}+A_{01}^{2} \mu_{1}^{2}\left(3-2 \mu_{1}\right)
\end{aligned}
$$

where $A_{i j}=r_{j} /\left(r_{j}-r_{i}\right), \mu_{i}=\left(1-\sqrt{r_{i} /\left(1+r_{i}\right)}\right) / 2, r_{0}$ and $r_{1}$ are given by (28) and (30), respectively.

For the MC DS/SFH system, only one of its carrier is overlaid by the NBI, with average SNR given by

$$
\begin{aligned}
r_{2}=\left\{2 K_{h} /(3 N)\right. & \\
& \left.+4\left[\frac{J / S \cdot U(1,-1)}{0.25 N_{T}}+\left(E_{b} / N_{0}\right)^{-1}\right]\right\}^{-1}
\end{aligned}
$$




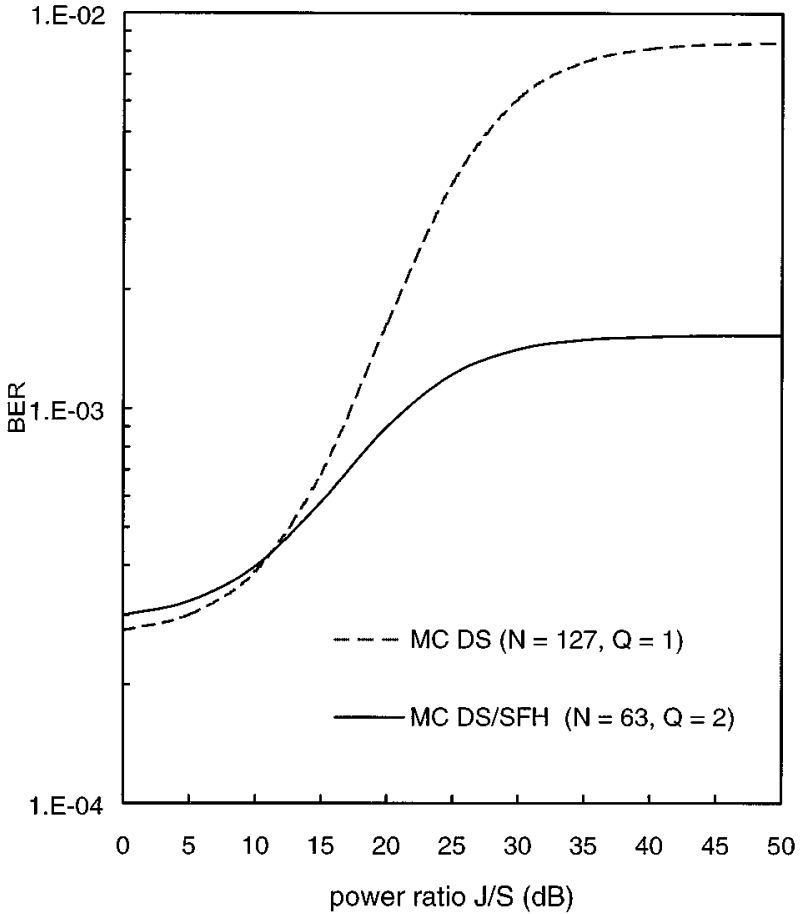

Fig. 6. BER performance versus interference power to signal power ratio $J / S\left(K=50, p=0.25, \delta=0, E_{b} / N_{0} \rightarrow \infty\right)$.

and the SNRs of the other three carriers free of interference are equal to $r_{0}$. Therefore, its $\left.P_{e}\right|_{K_{h}, q}$ take the formula given by (A12)

$$
\begin{aligned}
\left.P_{e}\right|_{K_{h}, q}= & A_{20} \mu_{0}+3 A_{20} A_{02} \mu_{0}^{2} \\
& +\left(6 A_{20} A_{02}^{2}-2 A_{20} A_{02}\right) \mu_{0}^{3} \\
& -8 A_{20} A_{02}^{2} \mu_{0}^{4}+3 A_{20} A_{02}^{2} \mu_{0}^{5}+A_{02}^{3} \mu_{2}
\end{aligned}
$$

where $A_{i j}=r_{j} /\left(r_{j}-r_{i}\right), \mu_{i}=\left(1-\sqrt{r_{i} /\left(1+r_{i}\right)}\right) / 2, r_{0}$ and $r_{2}$ are given in (28) and (32), respectively.

In Fig. 6, the BERs of both systems are plotted against $J / S$. It is seen that the MC DS/SFH system outperforms the MC DS system significantly. It is well known that diversity is essential to improve the performance. Since the affected carriers contribute little to the final decision, the MC DS/SFH system benefits from a higher "effective" diversity order.

Fig. 7 plots the performance of the two systems for different interference bandwidth $(K=50, J / S=30 \mathrm{~dB}, \delta=0)$. It is shown that the MC DS/SFH system keeps its advantage as long as the interference is narrower than one subchannel $(p \leq 0.25)$ so that the interference can not overlay two carriers simultaneously. However, as $B_{J}$ exceeds $B_{M}$, its advantage diminishes rapidly because only two carriers remain unaffected in the worst case, the same as the MC DS system. Once the interference fully covers the two middle subchannels $(p=0.5)$, the MC DS/SFH system lost its advantages.

Fig. 8 illustrates the effect of the interference location on the performance of the two systems $(K=50, J / S=30 \mathrm{~dB}, p=$ $0.25)$. It is shown that the performance of all system varies for different interference center frequencies. The MC DS system is shown to outperform the MC DS/SFH system when the NBI is largely contained in one subchannel, because both systems have

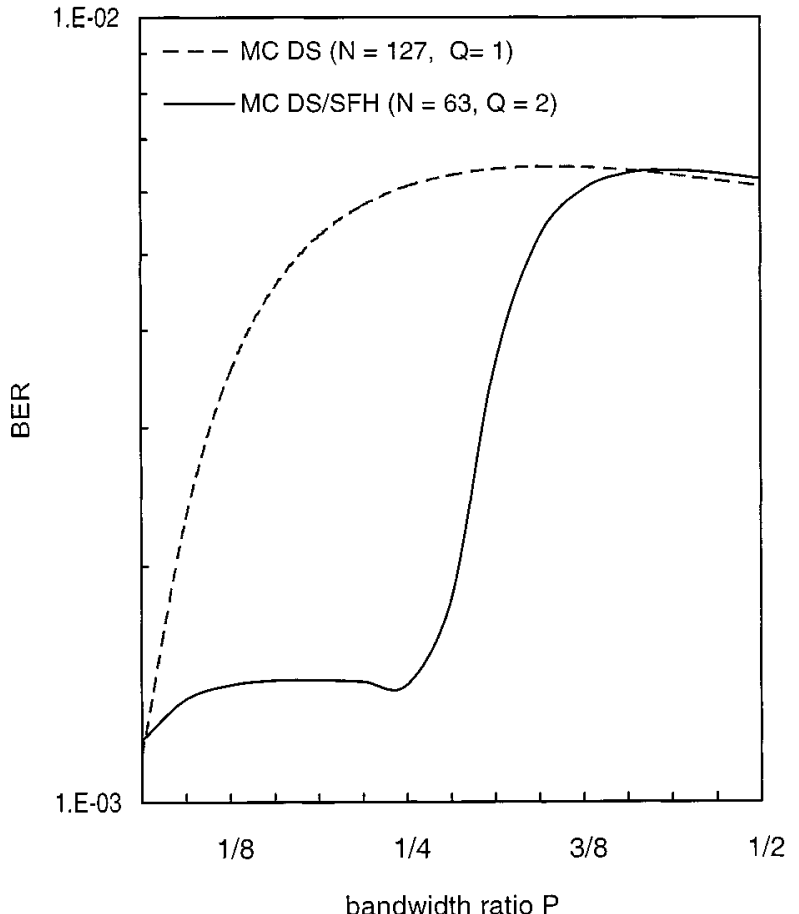

Fig. 7. BER performance versus interference bandwidth to system bandwidth ratio $p\left(K=50, J / S=30 \mathrm{~dB}, E_{b} / N_{0} \rightarrow \infty\right)$.

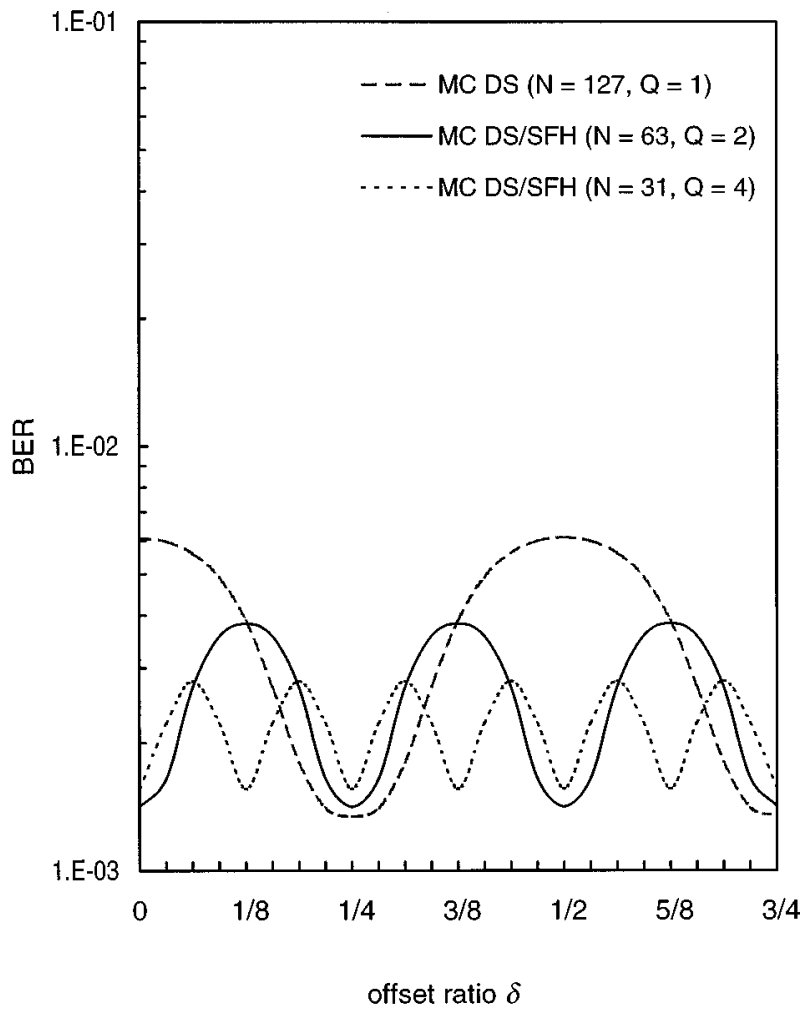

Fig. 8. Asymptotic BER with respect to central frequency offset ratio ( $K=$ $\left.50, J / S=30 \mathrm{~dB}, B_{J}=B_{M}, E_{b} / N_{0} \rightarrow \infty\right)$.

the same number of "clean" carriers. However, the performance of the MC DS/SFH system is more robust with respect to the variations of interference location, especially when the number of hopping frequencies is large $(Q=4)$. 


\section{CONCLUSION}

The MC DS/SFH-CDMA system is investigated in a multipath Rayleigh fading additive white Gaussian noise (AWGN) channel, with or without NBI. Its performance is compared with that of the MC DS-CDMA system. The result shows that the MC DS/SFH system, with a lower chip rate, can achieve approximately the same performance as the MC DS system in MAI suppression. However, it offers better and more robust performance than the MC DS system in the presence of NBI, especially when the interference bandwidth is narrower than one subchannel, which suggests that SFH technique can make a more efficient use of the unaffected bandwidth.

\section{APPENDIX A}

THE DeRIVATION OF $\left.P_{e}\right|_{K_{h}, q}$

Since $\beta_{i, m}$ is Rayleigh distributed, the signal to noise ratio $\left.\gamma_{m}\right|_{\beta_{i, m}, K_{h}, q}$ in (21) has a chi-square distribution, conditioned on $K_{h}$ and $q$. Its characteristic function is given by

$$
\psi_{m}(j u)=\frac{1}{1-\left.j u \bar{\gamma}_{m}\right|_{K_{h}, q}}
$$

where $\left.\bar{\gamma}_{m}\right|_{K_{h}, q}$ is the mean of $\left.\gamma_{m}\right|_{\beta_{i, m}, K_{h}, q}$ over $\beta_{i, m}$ and given by

$$
\begin{aligned}
\left.\bar{\gamma}_{m}\right|_{K_{h}, q}=\left\{2 K_{h} /(3 N)\right. & \\
& \left.+M\left[J / S \cdot U(m, q)+\left(E_{b} / N_{0}\right)^{-1}\right]\right\}^{-1} .
\end{aligned}
$$

Because $\left\{\beta_{i, m}\right\}$ are mutually independent, the characteristic function of $\left.\gamma_{b}\right|_{\left\{\beta_{i, m}\right\}, K_{h}, q}$ is simply the product of all $\psi_{m}(j u)$, i.e.,

$$
\psi_{b}(j u)=\prod_{m=0}^{M-1} \frac{1}{1-\left.j u \bar{\gamma}_{m}\right|_{K_{h}, q}} .
$$

The probability density function, $f_{\gamma}\left(\gamma_{b} \mid K_{h}, q\right)$, of $\gamma_{b}$ conditioned on $K_{h}$ and $q$, can be obtained by inverse Fourier transform of $\psi_{b}(j u)$, and $\left.P_{e}\right|_{K_{h}, q}$ is given by

$$
\left.P_{e}\right|_{K_{h}, q}=\int_{0}^{\infty} P_{e}\left(\gamma_{b}\right) f_{\gamma}\left(\gamma_{b} \mid K_{h}, q\right) d \gamma_{b}
$$

where $P_{e}\left(\gamma_{b}\right)$ is given by (25).

When all diversity branches have the same average SNR equal to $r$, i.e., $\psi_{b}(j u)=\left(1 /(1-j u r)^{M}\right),\left.P_{e}\right|_{K_{h}, q}$ has a closed form solution [7]

$$
\left.P_{e}\right|_{K_{h}, q}=P(M, r)=\mu^{M} \sum_{i=0}^{M-1}\left(\begin{array}{c}
M-1+i \\
i
\end{array}\right)(1-\mu)^{i}
$$

where $\mu=\lfloor 1-\sqrt{r /(1+r)}\rfloor / 2$.

When the SNRs of different branches are not identical (in the presence of NBI), $\psi_{b}(j u)$ can be expressed by a partial fraction expansion, i.e.,

$$
\psi_{b}(j u)=\frac{1}{\prod_{i}\left(1-j u r_{i}\right)^{D_{i}}}=\sum_{i} \sum_{j=1}^{D_{i}} \frac{B_{i, j}}{\left(1-j u r_{i}\right)^{j}}
$$

where $\sum_{i} D_{i}=M$ and

$$
\begin{aligned}
& B_{i, j}=\frac{\left(-r_{i}\right)^{\left(j-D_{i}\right)}}{\left(D_{i}-j\right) !} \\
& \quad \times\left.\left\{\frac{d^{\left(D_{i}-j\right)}}{d x^{\left(D_{i}-j\right)}}\left[\left(1-r_{i} x\right)^{D_{i}} \psi_{b}(x)\right]\right\}\right|_{x=1 / r_{i}}
\end{aligned}
$$

where $\left(d^{k} / d x^{k}\right)[f(x)]$ represents the $k$ th derivative of $f(x)$. Since the inverse Fourier transform and integration are linear, the corresponding probability of bit error can be written as

$$
\left.P_{e}\right|_{K_{h}, q}=\sum_{i} \sum_{d=1}^{D_{i}} B_{i, d} P\left(d, r_{i}\right) .
$$

For example, when $M=4$, two branches have SNR $r_{0}$ and the other two branches have SNR $r_{1}$ (the case we meet in the numerical section)

$$
\psi_{b}(j u)=\frac{1}{\left(1-j u r_{0}\right)^{2}\left(1-j u r_{1}\right)^{2}}
$$

one has

$$
\begin{aligned}
\left.P_{e}\right|_{K_{h}, q}= & A_{10}^{2} P\left(2, r_{0}\right)+2 A_{10}^{2} A_{01} P\left(1, r_{0}\right) \\
& +2 A_{01}^{2} A_{10} P\left(1, r_{1}\right)+A_{01}^{2} P\left(2, r_{1}\right) \\
= & A_{10}^{2} \mu_{0}^{2}\left(3-2 \mu_{0}\right)+2 A_{10}^{2} A_{01} \mu_{0}+2 A_{01}^{2} A_{10} \mu_{1} \\
& +A_{01}^{2} \mu_{1}^{2}\left(3-2 \mu_{1}\right)
\end{aligned}
$$

where $A_{i j}=r_{j} /\left(r_{j}-r_{i}\right)\left(i \neq j, r i \neq r_{j}\right), \mu_{i}=\lfloor 1-$ $\left.\sqrt{r_{i} /\left(1+r_{i}\right)}\right\rfloor / 2$.

When three branches have average SNR $r_{0}$ and the left one has average $\mathrm{SNR} r_{1}$, i.e.,

$$
\psi_{b}(j u)=\frac{1}{\left(1-j u r_{0}\right)^{3}\left(1-j u r_{1}\right)}
$$

one has

$$
\begin{aligned}
\left.P_{e}\right|_{K_{h}, q} & \\
= & A_{10} \sum_{d=1}^{3} A_{01}^{d-1} P\left(d, r_{0}\right)+A_{01}^{3} P\left(1, r_{1}\right) \\
= & A_{10} \mu_{0}+3 A_{10} A_{01} \mu_{0}^{2}+\left(6 A_{10} A_{01}^{2}-2 A_{10} A_{01}\right) \mu_{0}^{3} \\
& -8 A_{10} A_{01}^{2} \mu_{0}^{4}+3 A_{10} A_{01}^{2} \mu_{0}^{5}+A_{01}^{3} \mu_{1}
\end{aligned}
$$

where $A_{i j}=r_{j} /\left(r_{j}-r_{i}\right)\left(i \neq j, r_{i} \neq r_{j}\right), \mu_{i}=\lfloor 1-$ $\left.\sqrt{r_{i} /\left(1+r_{i}\right)}\right\rfloor / 2$.

\section{APPENDIX B}

\section{The Monte Carlo Simulation Model}

For rectangular chips and $0 \leq n T_{c} \leq \tau \leq(n+1) T_{c} \leq T$, as shown in [8], the partial crosscorrelation functions $R_{k i}$ and $\hat{R}_{k i}(\tau)$ are given by

$$
\begin{aligned}
R_{k i}(\tau)= & C_{k i}(n-N) T_{c}+\left[C_{k i}(n+1-N)\right. \\
& \left.-C_{k i}(n-N)\right]\left(\tau-n T_{c}\right) \\
\hat{R}_{k i}(\tau)= & C_{k i}(n) T_{c}+\left[C_{k i}(n+1)-C_{k i}(n)\right]\left(\tau-n T_{c}\right)
\end{aligned}
$$


where the discrete aperiodic cross-correlation term $C_{k i}(\cdot)$ is defined as

$$
C_{k i}(n)= \begin{cases}\sum_{j=0}^{N-1-n} a_{k}^{(j)} a_{i}^{(j+n)} & 0 \leq n \leq N-1 \\ \sum_{j=0}^{N-1+n} a_{k}^{(j-n)} a_{i}^{(j)} & -(N-1) \leq n \leq 0 \\ 0 & \text { else. }\end{cases}
$$

Once the code sequences are obtained, $R_{k i}(\tau)$ and $\hat{R}_{k i}(\tau)$ are determined by $\tau$. Note that $I_{k, m}$ in (19) is a zero-mean Gaussian random variable if $\tau_{k}$ is given, which follows from the fact that $\beta_{k, m} \cos \left[\Phi_{k, m}-\Phi_{i, m}\right]$ is a zero-mean Gaussian variable with variance $\rho$, therefore, $b_{k}^{(\lambda-1)} \beta_{k, m} \cos \left[\Phi_{k, m}-\Phi_{i, m}\right]$ and $b_{k}^{(\lambda)} \beta_{k, m} \cos \left[\Phi_{k, m}-\Phi_{i, m}\right]$ are also zero-mean Gaussian variables with the same variance. The conditional variance of $I_{k, m}$ is given by

$$
\sigma_{k}^{2}=2 \rho P\left\lfloor R_{k i}^{2}\left(\tau_{k}\right)+\hat{R}_{k i}^{2}\left(\tau_{k}\right)\right\rfloor .
$$

It is also obvious that $\left\{I_{k, m}\right\}$ are all uncorrelated since $E\left\{\cos \left[\Phi_{k_{1}, m}-\Phi_{k_{2}, m}\right]\right\}=0$ for $k_{1} \neq k_{2}$. Therefore, conditioned on $b_{i}^{(\lambda)}, \beta_{i, m},\left\{\tau_{k}\right\}, K_{h}$ and $q, \xi_{m}(\lambda)$ is a Gaussian random variable. The SNR of $\xi_{m}(\lambda)$ is defined as

$$
\begin{aligned}
& \left.\gamma_{m}\right|_{\beta_{i, m},\left\{\tau_{k}\right\}, K_{h}, q} \\
& \quad=\frac{E^{2}\left[\xi_{m}(\lambda) \mid \beta_{i, m}\right]}{2 \operatorname{Var}\left[\xi_{m}(\lambda) \mid\left\{\tau_{k}\right\}, K_{h}, q\right]} \\
& \quad=\frac{E_{c} T\left(\beta_{i, m}\right)^{2}}{2 \rho}\left[\sum_{k=1}^{K_{h}} \sigma_{k}^{2}+\sigma_{J}^{2}(m)+N_{0} T\right]^{-1} .
\end{aligned}
$$

The instantaneous SNR of $\xi(\lambda)$, conditioned on $\left\{\beta_{i, m}\right\},\left\{\tau_{k}\right\}, K_{h}$ and $q$, is given by

$$
\begin{aligned}
\left.\gamma_{b}\right|_{\left\{\beta_{i, m}\right\},\left\{\tau_{k}\right\}, K_{h}, q} & \left.\sum_{m=0}^{M-1} \gamma_{m}\right|_{\beta_{i, m},\left\{\tau_{k}\right\}, K_{h}, q} \\
= & \sum_{m=0}^{M-1} \frac{\left(\beta_{i, m}\right)^{2}}{2 \rho}\left\{T^{-2} \sum_{k=1}^{K_{h}}\left[R_{k i}^{2}\left(\tau_{k}\right)+\hat{R}_{k i}^{2}\left(\tau_{k}\right)\right]\right. \\
& \left.+M J / S \cdot X(m, q)+M\left(\frac{E_{b}}{N_{0}}\right)^{-1}\right\}^{-1} .
\end{aligned}
$$

To obtain the average BER $P_{e}$, the conditional BER is averaged over the statistics of $\gamma_{b}$, that is,

$$
P_{e}=\int_{0}^{\infty} P_{e}\left(\gamma_{b}\right) f_{\gamma}\left(\gamma_{b}\right) d \gamma_{b}
$$

where $f_{\gamma}\left(\gamma_{b}\right)$ represents the pdf of $\gamma_{b}$. It is difficult to get the analytical results, therefore, Monte Carlo integration over $\left\{\beta_{i, m}\right\},\left\{\tau_{k}\right\}, K_{h}$ and $q$ is performed to get $P_{e}$.

\section{REFERENCES}

[1] D. Lee and L. B. Milstein, "Comparison of multicarrier DS CDMA broadcast systems in a multipath fading channel," IEEE Trans. Commun., vol. 47, pp. 1897-1904, Dec. 1999.

[2] E. A. Sourour and M. Nakagawa, "Performance of orthogonal multicarrier CDMA in a multipath fading channel," IEEE Trans. Commun., vol. 44, pp. 356-367, Mar. 1996.

[3] S. Kondo and L. B. Milstein, "Performance of multicarrier DS CDMA systems," IEEE Trans. Commun., vol. COM-44, pp. 238-246, Feb. 1996.

[4] X. Gui and T. S. Ng, "Performance of asynchronous orthogonal multicarrier CDMA system in frequency selective fading channel," IEEE Trans. Commun., vol. 47, pp. 1084-1091, July 1999.

[5] Q. Chen, E. S. Sousa, and S. Pasupathy, "Multicarrier CDMA with adaptive frequency hopping for mobile radio systems," IEEE J. Select. Areas Commun., vol. 14, pp. 1852-1858, Dec. 1996.

[6] J. Wang and M. Moeneclaey, "Hybrid DS/SFH-SSMA with predetection diversity and coding for indoor radio multipath Rician fading channels," IEEE Trans. Commun., vol. 40, pp. 1654-1662, Oct. 1992.

[7] J. G. Proakis, Digital Communication, 3rd ed. New York: McGrawHill, 1995.

[8] M. B. Pursley, "Performance evaluation for phase-coded spread spectrum multiple access communication-Part I: System analysis," IEEE Trans. Commun., vol. 25, pp. 795-799, Aug. 1977.

[9] H. F. A. Roefs and M. B. Pursley, "Correlation parameters of random sequences and maximal length sequences for spread-spectrum multiple-access communications," IEEE Trans. Commun., vol. 27, pp. 1797-1604, Oct. 1979

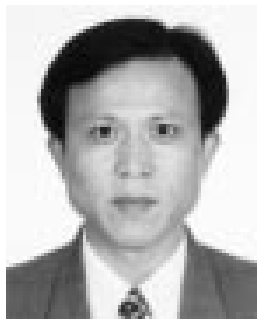

Jiangzhou Wang (M'91-SM'94) received the B.S. and M.S. degrees from Xidian University, Xian, China, in 1983 and 1985, respectively, and the Ph.D. degree (with Greatest Distinction) from the University of Ghent, Belgium, in 1990, all in electrical engineering.

From 1990 to 1992, he was a Postdoctoral Fellow in the University of California, San Diego, CA, where he worked on the research and development of cellular CDMA systems. From 1992 to 1995, he was a Senior System Engineer at Rockwell International Corporation, Newport Beach, CA, where he worked on the development an system design of wireless communications. Since 1995, he has been with the University of Hong Kong, Kowloon, Hong Kong, where he is currently an Associate Professor. He has held a Visiting Professor position in NTT DoCoMo, Japan. He was a Technical Chairman of IEEE Workshop in 3G Mobile Communications, 2000. He has published over one hundred papers, including more than 20 IEEE Transactions/Journal papers in the areas of wireless mobile and spread spectrum communications. He has written/edited two books, entitled "Broadband Wireless Communications" (Norwell, MA: Kluwer, 2001) and "3G Mobile Enhanced Technologies" (Norwood, MA: Artech House, 2002), respectively. He holds one U.S. patent in the GSM system. He is listed in Who's Who in the World.

Dr. Wang is an Editor for IEEE TRANSACTIONS ON COMMUNICATIONS and a Guest Editor for IEEE JOURNAL ON SeLECTED AREAS IN COMMUNICATIONS.

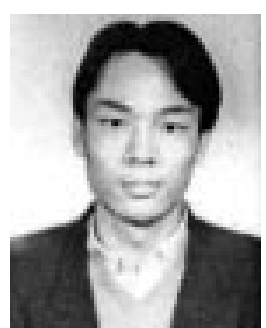

Hu Huang received the B.S. degree from the University of Science and Technology of China and the M.Phil. degree from the University of Hong Kong, Kowloon, Hong Kong, in 1997 and 2000, respectively, all in electrical engineering. Currently, he is working towards the Ph.D. degree in the Electrical and Computer Engineering Department, University of Maryland, College Park, MD 